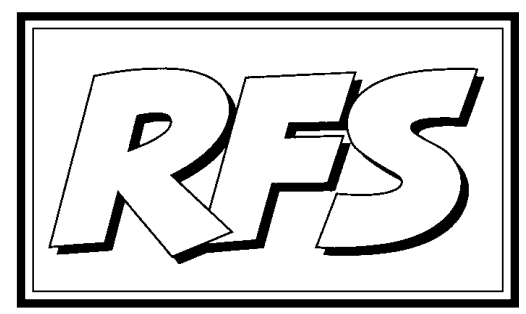

Revista de Fomento Social, 58 (2003), 343-363

\title{
Algunos problemas de la actividad financiera desde una perspectiva ética
}

\section{—Ma del Carmen LÓPEZ MARTín y Adolfo RODERO FRANGANILLO *- \\ (Palabras clave: Economía financiera, Mercados financieros, Ética. Key words: Financial Economics, Financial Markets, Ethical)}

\section{Introducción}

Los escándalos aparecidos en los últimos años en relación con la actividad empresarial en general y con las actuaciones financieras en particular, han creado un fuerte malestar entre los ciudadanos, unido a una gran desconfianza en relación con el mundo de las finanzas. Naturalmente que no se debe generalizar en este sentido, pero es evidente que se hace necesaria la existencia de limitaciones al funcionamiento de las entidades y de los mercados financieros.

En este escrito trataremos sobre todo de sistematizar estos problemas ya

* Facultad de CC. EE. y Empresariales - ETEA, adscrita a la Universidad de Córdoba. 
que no siempre tienen el mismo carácter des de un punto de vista moral. Así, en orden de más a menos gravedad, podemos distinguir:a) actuaciones delictivas; b) abusos que no se oponen claramente a las normas jurídicas; c) comportamientos rechazables desde un punto de vista ético; d) mal funcionamiento de las entidades o de los mercados; y e) defectos en el buen servicio a la clientela.

A partir de estos planteamientos, nuestro artículo contiene dos partes: 1을 sistematización de los comportamientos recientes de algunos de los componentes del sistema financiero siguiendo la clasificación anterior; 2 ) acciones emprendidas o propuestas para mejorar los mercados financieros en relación con los problemas detectados en la parte primera.

\section{Sistematización de las actuaciones criticables de las instituciones y de los mercados financieros}

\subsection{Posibles actuaciones delictivas}

Desde el inicio de la transición política, años setenta, han sido numerosos los casos de personas o entidades que han sido objeto de un proceso judicial. No es nuestro propósito limitarnos a aquellos casos en los que ha habido una sentencia firme; queremos aludir a todos los que han sido objeto de un proceso, aunque no se haya alcanzado aún una resolución o bien ésta haya sido absolutoria.

Entre los problemas más antiguos del período indicado, tenemos varios que afectaron a instituciones financieras: Sofico y los bancos del holding RUMASA fueron los más sonados. Más reciente es el proceso en relación con BANESTO que terminó con la condena de varios altos cargos. En este caso concreto, recordemos que el Banco de España decidió intervenir el banco debido a que los problemas detectad os en diversas inspecciones no habían sido corregidos: en el momento de la intervención se había encontrado un déficit de recursos propios superior a los 3.600 millones de euros (algo más de 600.000 millones de ptas.); una vez que se intervino el banco, se nombró un nuevo consejo de administración, encargado de su saneamiento ${ }^{1}$ y, más

1 El coste acumulado que ha supuesto el saneamiento del banco por parte del Fondo de Garantía de Depósitos (FGD) era (a diciembre de 2001) 4.687,4 millones de euros. De dicho total, hasta diciembre de 2001 se habían recuperado 3.519,4 millones, por lo tanto el coste del saneamiento a la fecha indicada ascendía a 1.168 millones de euros. 
tarde, el banco fue subastado y adquirido por el entonces Banco de Santander.

Si nos limitamos a los últimos años, hay que citar en primer lugar el expediente al BBVA en el que han participado la Audiencia Nacional, el Banco de España y la Comisión Nacional del Mercado de Valores (CNMV) y el FBI en Estados Unidos, cada uno dentro de la órbita de sus competencias. Las principales acusaciones son la existencia de cuentas ocultas en la isla de J ersey y en Liechtenstein, la creación de fondos de pensiones en beneficio de los altos cargos, blanqueo de dinero y sobornos a políticos latinoamericanos. El proceso no ha finalizado todavía pero ha dado ya lugar a la salida de un número elevado de altos cargos del banco y, sobre todo, ha creado un clima de desconfianza sobre el comportamiento de los grandes bancos.

Menos trascendencia ha tenido un auto dictado por la Audiencia Nacional contra el BSCH por la realización de cesiones de crédito a su clientela, operaciones de carácter fiscalmente opaco que podrían ser calificadas de delito fiscal, unido a la acusación de falsedad documental.

También hay que recordar el caso Gescartera, entidad de inversión financiera que ha ocasionado un grave perjuicio económico a su clientela, la cual en muchos casos ha perdido casi todo el dinero que había encomendado a esta agencia de valores para que lo invirtiese. Esta investigación está relacionada presuntamente con la apertura de cuentas opacas en Suiza por el importante banco británico HSBC que ha sido objeto de una multa del ministerio de Economía. Este escándalo ha llevado a la sustitución de la máxima responsable de la CNMV y ha tenido importantes repercusiones políticas.

Un fallo judicial en relación con una caja de ahorros ha sido la condena de la Caixa por unos contratos de seguro, denominados pensiones inmediatas, por no haber liquidado el impuesto sobre la renta.

También hay que citar las operaciones de blanqueo de dinero que se han visto facilitadas por la implantación del euro. Aunque la denuncia de las operaciones sospechosas por parte de las entidades de depósito va en aumento, también ha habido varias actuaciones que no han sido impedidas por las instituciones financieras.

La condena por estafa de los financieros Alberto Alcocer y Alberto Cortina, dictada en el año actual, aunque no se refiere a una entidad financiera, ha tenido también efectos sobre un banco, el Zaragozano, de 
cuyo Consejo han tenido que dimitir.

Evidentemente, los problemas no se limitan a España ya que también ha habido graves situaciones en otros países. No podemos entrar en detalles en este sentido, pero sí hay que subrayar que los procesos judiciales han alcanzado a entidades muy importantes, tales como el banco estadounidense J. P. Morgan y el alemán Deutsche Bank.

A partir de estos casos no se debe realizar una valoración descalificadora de todo el sistema, pero no cabe duda que son lo suficientemente graves para alarmar al ahorrador. Hay que tener en cuenta que, resumiendo, en los diferentes casos que se han comentado a lo largo de este epígrafe, las entidades se han visto afectadas por delitos (probados o presuntos) de tipo fiscal (contra la hacienda pública), falsedad documental, apropiación indebida, malversación de fondos o blanqueo de dinero.

\subsection{Abusos que no tienen claramente carácter delictivo}

Las actividades de las instituciones en los mercados financieros pueden en ocasiones perjudicar a sus clientes, bien porque tengan un carácter abusivo o bien porque se opongan a normas legales cuya vulneración no permite normalmente una actuación judicial.

Los abusos se producen con frecuencia porque las instituciones financieras tienen más poder que sus clientes, lo que les permite una situación de dominio que atenta contra la libre concurrencia o la competencia perfecta. En este sentido, hay que recordar que los problemas se han planteado sobre todo en relaciones con las comisiones bancarias; en la cuenta de resultados de las entidades de depósito se ha producido una reducción del margen de intermediación y un aumento de las comisiones, lo que unido a la poca transparencia de estos ingresos explica el que a veces se produzcan prácticas abusivas.

Las quejas más frecuentes respecto a esta cuestión se refieren a las comisiones cobradas a los establecimientos comerciales por el uso de tarjetas, la utilización de contratos con cláusulas genéricas, el cargo de comisiones no pactadas, las condiciones de las hipotecas, etc. Todo ello determina quejas y reclamaciones ante el Banco de España y la Organización de Consumidores y Usuarios, que en su mayor parte se dirigen contra la banca y en bastante menor medida contra las cajas de ahorros. 
En este punto, es preciso recordar que, en la actualidad, la cuantía de las comisiones cobradas por las entidades financieras a su clientela es libre; las entidades tienen la obligación de enviar al Banco de España, para su registro y aprobación, las comisiones y gastos repercutibles que, como máximo, cobrarán a la clientela. La única limitación que existe en las normas relativas a esta cuestión es que las tarifas cargadas a los clientes tienen que corresponder a servicios efectivamente prestados o a gastos habidos y que en ningún caso podrán cobrarse comisiones o gastos por servicios no aceptados 0 solicitados. El problema, por tanto, no está en las cantidades que se puedan cobrar, sino en si dichas cantidades pueden o no cobrarse en unas circunstancias concretas. Por otra parte, en todo caso, los clientes deben recibir la información completa acerca de estas tarifas y del resto de las condiciones que resulten de aplicación a los contratos que efectúan con las entidades de crédito ${ }^{2}$.

En cuanto a comportamientos que quizás vulneren normas pero que normalmente no terminan en los tribunales, distinguiríamos dos tipos de actuaciones:

1. Los conflictos de intereses. Las entidades, sobre todo de inversión, pueden aconsejar a sus clientes de forma poco objetiva para orientar la inversión hacia sus empresas clientes o hacia empresas filiales o que mantienen lazos económicos con la institución financiera. Estos casos, frecuentes en Estados Unidos, han terminado en multas o procesos a ciertos bancos de inversión, pero en general son difíciles de controlar; en nuestro país no ha habido demandas de este carácter, pero ciertamente el problema ha existido. La propia Ley del mercado de valores ha legislado sobre ello, pero su virtualidad es escasa.

2. La información privilegiada. La utilización de información confidencial que afectará al comportamiento futuro de unos valores concretos, antes de que sea pública, ha permitido obtener ganancias a unos agentes en perjuicio de los restantes agentes del mercado. Hay sospechas fundadas de casos de información privilegiada, que sin embargo no han podido ser probados, ni por lo tanto han llevado a una sentencia judicial.

2 Las normas relativas a la transparencia de las operaciones y protección de la clientela establecen en qué casos concretos es obligatoria la entrega de los contratos y de la información completa de las operaciones por parte de las entidades y cuándo se puede entender que esta entrega no es necesaria y basta con la información general que la entidad debe tener a disposición de todos sus clientes. 
También hay que citar las ofertas públicas de adquisición (OPA) que en su normativa actual permite que estas operaciones ofrezcan beneficios, incluso financieros, a los propietarios de grandes paquetes de acciones, en perjuicio de los pequeños accionistas que se ven privados de las plusvalías que puede proporcionar una OPA.

\subsection{Comportamientos poco éticos}

Nos planteamos el antiguo tema de si todo lo que es legal puede ser aceptado positivamente desde un punto de vista ético.

Las páginas de economía han recogido en los últimos tiempos comportamientos que pueden generar una valoración negativa de las instituciones financieras.

Señalamos en primer lugar las retribuciones millonarias de los consejeros de los bancos, especialmente en el momento de su baja en el Consejo de administración o de su jubilación. Algunos casos, algo menos ruidosos, se han producido en cajas de ahorros. Los pequeños accionistas se sorprenden de que estos pagos se produzcan en los momentos en los que las cuentas de resultados descienden y cuando las caídas del precio en la bolsa de estos valores están provocando importantes pérdidas. Algunas de estas retribuciones han llegado a ser investigadas por la justicia, pero, en general, insistimos en que no se trata, normalmente, de un problema de ilegalidad, sino de ética dudosa.

Muy próximo al tema anterior es el de las opciones sobre acciones (stock options), sistema a través del cual los directivos de las entidades tienen derecho a la compra de acciones a un precio determinado. Esta fórmula tiene poco coste para la entidad; la posible ganancia se obtiene por el alza, en su caso, de la cotización bursátil. El sistema no es, en principio, criticable; se han subrayado, sin embargo, las ganancias multimillonarias que han obtenido algunos directivos en el pasado. En algún caso, la existencia de estas opciones ha ido unida a una manipulación de las cuentas para obtener plusvalías, comportamiento claramente fraudulento. Por otra parte, la caída de la bolsa en los últimos años ha dejado sin contenido muchos programas de opciones sobre acciones. Muy recientemente han vuelto a ofrecerse algunas ofertas de opciones para directivos bancarios.

Esta problemática ligada a la retribución de los consejeros se ve enturbia- 
da por la falta de transparencia sobre el total de los pagos a cada uno de ellos. Esta información no se da a conocer en la mayor parte de las entidades.

Otro tema distinto es el de la especulación en los mercados financieros. Tradicionalmente se ha mantenido que la especulación tiene un papel positivo en los mercados: la estabilidad de los precios y la cobertura del riesgo. Sin rechazar totalmente esta valoración, hay que recordar que también existe una especulación que desestabiliza los mercados. En todo caso, un volumen muy elevado de especulación puede alejar a los mercados financieros de su función básica: la financiación de la actividad real. En este sentido se puede citar que el $99 \%$ de las operaciones en los mercados de divisas tienen una finalidad financiera, con un componente elevado de especulación. Estas actividades tienen efectos muy negativos en los mercados que, en algunos casos, pueden llevar a la caída de ciertas divisas o a comportamientos erráticos de los mercados de valores ${ }^{3}$. Cuando el especulador, como ha sucedido en ciertos casos, utiliza los mercados a plazo o a crédito para influir negativamente sobre el precio de un valor, su actuación debe ser juzgada de forma muy negativa.

Un último ejemplo es el de la utilización incorrecta de los derivados financieros: la ocultación del riesgo y la contabilización de los resultados periódicos de estas operaciones como beneficio cierto constituyen un nuevo caso que ha sacudido a las bolsas, sobre todo a las estadounidenses.

\subsection{El buen o el mal funcionamiento del sistema financiero}

La función básica del sistema financiero es la de asegurar la utilización óptima de los recursos financieros 0 , en otros términos, la financiación más adecuada de la actividad real. El buen funcionamiento del sistema es un requisito prioritario de la actividad económica.

Este punto de partida tiene dos vertientes:

3 En este punto resulta muy ilustrativa la crisis vivida por los mercados de divisas en los meses de agosto a noviembre de 1992, que originó la salida de la libra esterlina y la lira italiana del mecanismo de cambios del Sistema Monetario Europeo y dos devaluaciones sucesivas de la peseta, en los meses de septiembre y noviembre, respectivamente. Los ataques especulativos que sufrieron las monedas citadas a lo largo de estos meses hicieron que los bancos centrales tuvieran que intervenir activamente en los mercados de divisas para frenar la depreciación de las cotizaciones (el volumen de reservas centrales en España disminuyó entre septiembre y noviembre de dicho año más de 23.700 millones de dólares, casi 2,5 billones de ptas.). 
1) Existe un problema de liquidez. La falta de liquidez de las instituciones puede conducir a una crisis del sistema.

2) Hay un segundo problema que es la solvencia. La existencia de un volumen adecuado de recursos propios es una condición necesaria para asegurar la solvencia.

La crisis del sistema financiero español, que comenzó principalmente en los años setenta, tuvo repercusiones judiciales en muchos casos, pero mucho más general fue el problema de un mal funcionamiento de las entidades por defectos de liquidez y de solvencia. Si consideramos los datos correspondientes al período 1977-85, la crisis afectó, en mayor o menor medida, a 56 bancos y a 23 empresas bancarias, que representaban aproximadamente el $27 \%$ de los recursos propios, de los recursos ajenos y de los trabajadores de los bancos españoles en esas fechas ${ }^{4}$. Una información adicional que puede completar la anterior es la recogida en el cuadro 1; en dicho cuadro se expone una estimación de los costes que supuso la crisis bancaria en los años citados, los cuales comprenden no sólo las aportaciones realizadas por el sector público para el saneamiento de las entidades afectadas por la crisis, sino también los recursos en los que contribuyó el sector privado 5 .

Medidas anteriores o posteriores a la crisis han tratado de eliminar estos problemas: coeficiente de caja, Fondos de Garantía de Depósitos, coeficiente de solvencia. Actualmente se mantiene que los conglomerados financieros (entidades que realizan actividades bancarias, de seguros y de fondos de inversión), necesitan unos niveles de solvencia más elevados y unos controles más estrictos.

Centrándonos momentáneamente en los fondos de garantía de depósitos, cabe recordar aquí que, para cada uno de los tres tipos de entidades que en España pueden captar depósitos (bancos, cajas de ahorro y cooperativas de crédito), se creó después de la crisis bancaria, un FGD cuya función principal

4 A. Cuervo (1987), p. 23

5 Como contribución del sector público al saneamiento de las entidades afectadas por la crisis bancaria se han considerado los recursos aportados por el Banco de España al FGD, las ayudas del Estado a los bancos del grupo Rumasa y también el costequesupuso para el Banco de España la concesión de créditos blandos a todas estas instituciones. Por su parte, el coste para el sector privado ha sido estimado a partir de los fondos entregados al FGD y los entregados directamente a las entidades afectadas para su saneamiento. 
es garantizar a los depositantes que recuperarán los recursos entregados en caso de crisis de la entidad en la que han depositado los fondos ${ }^{6}$. Además de ello, estos tres fondos también se encargan de realizar el saneamiento de las instituciones que, en su caso, tengan dificultades de solvencia ${ }^{7}$. Precisamente, en el cuadro 1, como complemento a la información sobre los costes que ha supuesto la crisis bancaria que se produjo entre 1977 y 1985, también se ha incluido cuál ha sido el coste que ha supuesto para los tres fondos de garantía de depósitos los saneamientos que han efectuado en las entidades en las que han intervenido.

CUADRO 1

Costes de la crisis bancaria y de los saneamientos efectuados por los fondos de garantía de depósitos

Importes

Coste de la crisis bancaria producida entre 1977 y 1985 $1.580 .836^{*}$

Aportaciones públicas (FGD, ayudas del Estado a Rumasa; coste para el Banco de España) $1.215 .727^{*}$

Aportaciones sector privado (FGD, aportaciones directas a saneamientos) 365.109*

Coste de los saneamientos efectuados por los fondos de

garantía de depósitos $522.036 * *$

FGD en establecimientos bancarios $492.219,7^{* *}$

FGD en cajas de ahorro $26.239,0 * *$

FGD en cooperativas de crédito $3.577,3^{* *}$

(*) Datos en millones de pesetas constantes de 1985.

(**) Datos acumulados en millones de ptas. a 31-12-2001.

Fuente: A. Cuervo (1987), p. 191 y LA CaIXA (2002), Informe mensual julio-agosto, p. 74.

6 En la actualidad, la cantidad garantizada asciende a 20.000 euros por persona.

7 Por ejemplo, en el caso de BANESTO, cuando el banco fue intervenido por el Banco de España, el FGD en establecimientos bancarios fue el encargado de su saneamiento, para lo cual nombró a un nuevo consejo de administración. 
Otro aspecto importante es si las entidades financieras deben invertir directamente en empresas o no deben hacerlo, siempre desde el punto de vista de la financiación de la actividad real. Se podría recordar que durante la crisis económica de los años setenta la banca eliminó sus inversiones empresariales, acentuando así los problemas de la economía española. Mucho más reciente es la participación de las entidades de depósito en las empresas, sobre todo en las de mayor importancia. Esta política parece adecuada, si bien puede llevar a la concentración del poder económico, lo que tiene riesgos evidentes.

Las fusiones entre entidades financieras tienen en principio una finalidad de mejorar la productividad de aquéllas. Sin embargo, en muchos casos han tenido una finalidad defensiva. La mayor dimensión de las entidades parece necesaria en un ámbito europeo o mundial, pero también llevan a una situación de oligopolio que, repetimos, tiene riesgos para la economía real.

Relacionado indirectamente con el contenido de este apartado está el aspecto de la valoración de los resultados bancarios. Prescindiendo de las posibles manipulaciones, hay que considerar que las normas contables europeas son menos estrictas que las estadounidenses, lo que determina cifras distintas de resultados en ambos espacios. Puesto que la contabilización de los resultados es una de las medidas del funcionamiento de las entidades, estas divergencias enturbian la apreciación del inversor y, en último caso, del mercado.

\subsection{El servicio a la clientela}

Aunque el título de este apartado va ligado directamente a la actividad de las instituciones bancarias, pretendemos aplicarlo a los mercados financieros en general, aludiendo a dos vertientes de las operaciones financieras: el destinatario de la financiación (demanda de recursos) y los aportantes de fondos (oferentes de recursos).

En relación con la demanda de financiación, no siempre las operaciones de las entidades de depósito responden a las necesidades de su clientela de activo. Aspectos tales como el plazo, las garantías y la localización no siempre responden a los requerimientos de los clientes. Así, las dificultades de las PYMES para obtener créditos, la falta de atención a algunos sectores (por ejemplo, el sector agrario), la concentración de los préstamos en los hipotecarios, la falta de atención al desarrollo regional, etc., son algunos 
ejemplos de ineficiencia de las entidades; ello ha determinado en distintos momentos la actividad de la banca pública (ya desaparecida), los convenios del sistema con los organismos públicos y la creación de otras instituciones financieras que cubren algunos de estos problemas a cambio de un mayor coste y de una elevación del riesgo.

Respecto a la oferta de fondos, es decir, desde el punto de vista del ahorrador, los dos aspectos importantes son la seguridad y la rentabilidad. Los problemas de inseguridad no son frecuentes, sólo en los casos más graves (RumAsa, BANESTo, GesCaRTeRA), se han producido pérdidas importantes delas inversiones ${ }^{8}$; la búsqueda, sin embargo, de la rentabilidad puede llevar a inversiones de alto riesgo (los llamados hedge funds), muy populares en Estados Unidos, que suelen ir unidos a inversiones en paraísos fiscales y en algunos casos a un auténtico fraude. Además, no hay que olvidar que, en general, la información ofrecida al cliente es incompleta u oscura, hay que acudir a los folletos completos para conocer en detalle las condiciones de las ofertas; estos folletos, que suelen requerir conocimientos técnicos, no se suelen facilitar espontáneamente a los posibles inversores.

La rentabilidad del ahorro ha sufrido variaciones muy elevadas durante los últimos años. Las épocas de subidas de las bolsas y de los tipos de interés han proporcionado altos rendimientos a los ahorradores, mientras que la crisis de los mercados de valores y los bajos tipos de interés, como sucede en la actualidad, reducen los rendimientos y no llegan siquiera en muchos casos a asegurar el capital invertido. Esto es especialmente grave en los fondos de pensiones y en fondos de inversión, en los que aparecen rentabilidades negativas, mientras que las sociedades gestoras y depositarias obtienen sustanciosas comisiones. No hay que olvidar que el pequeño ahorro sólo percibe una parte poco elevada de las plusvalías, mientras que la mayor parte de éstas van a los grandes inversores y a las instituciones financieras.

8 Por ejemplo, en el caso de BAnesto, justo antes de la intervención del Banco de España, las acciones del banco cotizaban a un valor de 1995 ptas. y, tras el periodo de suspensión en la cotización, pasaron a tener un valor de unas 700 ptas. Por lo tanto, cada acción perdió casi dos terceras partes de su valor; en otras palabras, el valor de las inversiones de los accionistas pasó en apenas un mes y medio a ser de un tercio del que tenían en diciembre de 1993.

Más recientemente, durante 2001, las cantidades reclamadas por las personas y organizaciones que habían confiado recursos a GeSCARTERA superaban los 15.000 millones de pesetas ( 94 millones de euros). 


\section{Medidas correctoras de los mercados financieros}

Es evidente que un panorama tan negativo no podía por menos que determinar acciones para corregir en los posible estas situaciones. Estas acciones han sido tomadas desde diversos ámbitos, las autoridades, las propias entidades o los mismos clientes afectados por los problemas comentados. Intentando sistematizar las diversas iniciativas adoptadas, las comentamos a continuación encuadrándolas en tres grandes bloques: las impulsadas por las autoridades, las relacionadas con las pautas de buen gobierno y las que tienen su origen en el sector privado, ya sea en las entidades y mercados o bien como resultado de la iniciativa de los usuarios financieros (en sentido amplio).

\subsection{Las iniciativas impulsadas por las autoridades}

En este punto deben considerarse, en principio, las iniciativas adoptadas desde el ámbito regulador, con el objeto de establecer normas que tratan de evitar algunos de los problemas que hemos comentado anteriormente. En este sentido ya hemos citado previamente algunas medidas que tratan de asegurar la solvencia y liquidez de las entidades: coeficiente de caja, coeficiente de solvencia y Fondo de Garantía de Depósitos, a las que habría que añadir el Fondo de Garantía de Inversiones; éste, si bien estaba previsto en la ley de reforma del mercado de valores del año 1998, fue creado efectivamente a raíz del caso Gescartera.

Los casos más graves han sido objeto de procesos judiciales o de actuaciones administrativas. El delito económico estaba casi ausente de las normas jurídicas y, sobre todo, no era frecuente la aplicación de las escasas normas existentes. Durante los últimos veinticinco años esta realidad ha variado profundamente: procesos judiciales muy conocidos, reforma del código penal (si bien con un límite al fraude fiscal superior al establecido en el convenio de la Unión Europea), persecución del blanqueo de dinero por parte del Ministerio de Hacienda (que ha exigido información a los bancos sobre sus cuentas de depósito) y persecución de los fraudes contables.

A medio camino entre la norma imperativa y la autorregulación, se encuentran las limitaciones y recomendaciones de la ley financiera sobre el denominado abuso de mercado (que se refiere principalmente a la información privilegiada y a la manipulación de las cotizaciones) y sobre los conflictos de intereses; este tratamiento tiene antecedentes en las leyes del 
mercado de valores, donde entre otras propuestas, se recomendaban las denominadas murallas chinas, es decir, la separación de los departamentos de análisis y de inversión de las entidades de depósito. Las innovaciones introducidas por la ley financiera han actualizado, completado y desarrollado la regulación sobre estos aspectos tomando como referencia las líneas básicas de la normativa comunitaria sobre los mismos ${ }^{9}$.

Así, los principales cambios introducidos han supuesto la extensión del ámbito de aplicación de la información privilegiada tanto en lo referente a los valores como a los mercados a todos los instrumentos financieros y sistemas organizados de contratación, el desarrollo detallado de la información que debe comunicarse al mercado de forma inmediata, la especificación de medidas preventivas del uso de información privilegiada relativas a la organización de las entidades que prestan servicios en los mercados; además se ha recogido de forma más explícita y pormenorizada la infracción de manipulación de las cotizaciones y, para permitir la aplicación más efectiva de las medidas adoptadas, se han reforzado las competencias de la CNMV. Aunque no cabe duda de que estas iniciativas redundan en un mejor funcionamiento de los mercados, no podemos olvidar que estos comportamientos no son fácilmente detectables por lo que es indispensable la colaboración de las entidades para su erradicación.

Paradójicamente los dos máximos responsables de los organismos supervisores: Banco de España y CNMV, han alertado del peligro de regular en exceso a los mercados e instituciones financieras.

La inquietud de los inversores ha dado lugar a la creación de figuras tales como el protector del inversor en la bolsa de Madrid, el Servicio de reclamaciones del Banco de España, el departamento de atención al público de la CNMV, etc. Estas figuras, en el caso de los organismos supervisores (Banco de España, CNMV y Dirección General de Seguros), también se han visto modificadas recientemente por la ley financiera. En ella, se regula la figura del Comisionado para la defensa del Cliente de Servicios financieros, que se concreta en la creación de tres órganos para defensa de los clientes de servicios bancarios, de los inversores y de los asegurados y partícipes en planes de pensiones, dependientes de los tres organismos supervisores, manteniendo el derecho de

9 Esta normativa está contenida en la directiva del Parlamento europeo y del Consejo relativa a las operaciones con información privilegiada y la manipulación del mercado, de reciente aprobación; tras la misma, el Comité de Reguladores de Valores Europeos debía proponer las medidas de ejecución de los principios recogidos en la directiva. 
Ios clientes a acudir a otros sistemas de protección ${ }^{10}$.

En relación con estas tres figuras, aunque hayan visto modificada en parte su regulación, vienen realizando labores de protección de los intereses de los clientes de las instituciones financieras (en algún caso desde hace bastante tiempo ${ }^{11}$ ); sin embargo, sobre ellas se han realizado críticas en el sentido de que, hasta ahora, la normativa que las regula no obliga a que las entidades sobre las que se hace la reclamación deban cumplir obligatoriamente el contenido de los informes de estos servicios y sus conclusiones. A pesar de este defecto legal, generalmente el cumplimiento de los criterios de dichos informes ha sido elevado y las entidades han rectificado de forma mayoritaria las actuaciones sobre las que se les ha reclamado en el sentido en el que se manifestaban los servicios mencionados.

\section{CUADRO 2}

\section{Distribución del contenido de las reclamaciones a la CNMV (no incluye 1394 relacionadas con Gescartera) y al Banco de España en 2001}

\begin{tabular}{|l|r|r|l|r|r|}
\hline \multicolumn{3}{|c|}{ Reclamaciones ante la CNMV } & \multicolumn{3}{c|}{ Reclamaciones ante el Banco de España } \\
\hline Asunto & Núm. & \% & Asunto & Núm. & $\%$ \\
\hline Emisiones de valores & 225 & 16,2 & Operaciones activas & 768 & 20,8 \\
\hline OPA & 77 & 5,6 & Operaciones pasivas & 935 & 25,4 \\
\hline Acontecimientos de la vida societaria & 52 & 3,8 & Cuestiones sobre valores y seguros & 791 & 21,5 \\
\hline Fondos de Inversión & 342 & 24,7 & Letras y cheques & 275 & 7,5 \\
\hline Operaciones con valores & 469 & 33,9 & Deuda del Estado & 16 & 0,4 \\
\hline Cotizaciones, frecuencia, liquidez, etc. & 22 & 1,6 & Divisas y billetes extranjeros & 58 & 1,6 \\
\hline Gestión de carteras & 39 & 2,8 & Tarjetas de crédito & 348 & 9,4 \\
\hline Entidades no registradas & 104 & 7,5 & Reclamaciones varias & 262 & 7,1 \\
\hline & & & Transferencias & 102 & 2,8 \\
\hline Otros & 55 & 4,0 & Otros & 6 & 0,2 \\
\hline Total & $\mathbf{1 . 3 8 5}$ & & Total & $\mathbf{3 . 5 6 1}$ & \\
\hline
\end{tabular}

Fuente: CNMV (2002), Informe anual sobre reclamaciones 2001, p. 18 y BANCO DE EsPAÑA (2002), Memoria del Servicio de Reclamaciones 2001, p. 26.

10 Se aplica el principio de ventanilla única en el ejercicio de la reclamación por los clientes, que pueden dirigirse a cualquier comisionado.

11 En concreto, el Servicio de reclamaciones del Banco de España se creó por la orden de 3 de marzo de 1987 y comenzó su actividad el 1 de septiembre de dicho año. 
A título de ejemplo, el cuadro 2 muestra el contenido de las reclamaciones efectuadas ante la CNMV y ante el Banco de España, así como los asuntos sobre los que se realizaban las mismas. En este cuadro se aprecia que son más numerosas las reclamaciones efectuadas ante el Banco de España, aunque en el año 2001, las presentadas ante la CNMV experimentaron un fuerte aumento como consecuencia de las relacionadas con el caso GESCARTERA. La información de este cuadro se completa con la que aparece en los cuadros 3 y 4 , los cuales muestran el resultado de estas reclamaciones. Como se puede apreciar en ellos, al go menos de la quinta parte delas reclamaciones efectuadas han dado lugar a un informe favorable al demandante, mientras que un porcentaje similar, aunque ligeramente superior, eran favorables a las entidades.

CUADRO 3

\section{Resultados de las reclamaciones presentadas por los inversores ante la CNMV contra empresas sometidas a su supervisión}

\begin{tabular}{|l|r|r|r|r|}
\hline \multirow{2}{*}{ Clasificación de las reclamaciones } & \multicolumn{2}{|c|}{ Número } & \multicolumn{2}{|c|}{$\%$} \\
\cline { 2 - 5 } & $\mathbf{2 0 0 0}$ & $\mathbf{2 0 0 1}$ & $\mathbf{2 0 0 0}$ & $\mathbf{2 0 0 1}$ \\
\hline Reclamaciones con pronunciamiento y/o resolución & $\mathbf{7 3 5}$ & $\mathbf{7 2 4}$ & $\mathbf{6 3}$ & $\mathbf{5 2}$ \\
\hline Avenimiento / Allanamiento (1) (Acuerdos) & 167 & 133 & 14 & 9 \\
\hline Con informe favorable(2) & 161 & 258 & 14 & 19 \\
\hline Con informe desfavorable(3) & 400 & 325 & 34 & 23 \\
\hline Desistimiento (4) & 7 & 8 & 1 & 1 \\
\hline Reclamaciones que no precisan pronunciamiento & $\mathbf{4 2 4}$ & $\mathbf{6 6 1}$ & $\mathbf{3 6}$ & $\mathbf{4 8}$ \\
\hline Caducidad (5) & 15 & 13 & 1 & 1 \\
\hline Con información al reclamante(6) & 389 & 608 & 33 & 44 \\
\hline Fuera de competencias dela CNMV(7) & 20 & 40 & 2 & 3 \\
\hline Pendientes & $\mathbf{1 0}$ & $\mathbf{0}$ & $\mathbf{1}$ & $\mathbf{0}$ \\
\hline T0TAL & $\mathbf{1 . 1 6 9}$ & $\mathbf{1 . 3 8 5 *}$ & $\mathbf{1 0 0}$ & $\mathbf{1 0 0}$ \\
\hline
\end{tabular}

* no incluye 1394 correspondientes a Gescartera

(1) Avenimiento / Allanamiento: Las partes llegan a un acuerdo; (2) Informe favorable: El reclamante tiene razón; (3) Informe desfavorable: El reclamante no tiene razón; (4) Desistimiento: El cliente retira su queja; (5) Caducidad: La reclamación se realiza de forma anónima o sin dirección, lo que impide su tramitación; (6) Información al reclamante: La información que la CNMV trasmite al reclamante resuelve la incidencia; (7) Fuera de competencias de la CNMV: La reclamación es competencia de otros organismos.

Fuente: CNMV (2002), Informe anual sobre reclamaciones 2001, p. 16. 


\section{CUADRO 4 \\ Resultados de las reclamaciones archivadas por el Banco de España en 2001}

\begin{tabular}{|l|r|r|}
\hline Clasificación de las reclamaciones & Núm. & $\%$ \\
\hline Sin solicitud de alegaciones & $\mathbf{1 . 2 6 4}$ & $\mathbf{4 1 , 4}$ \\
\hline El reclamante no aportó la documentación solicitada & 178 & 5,8 \\
\hline Solicitudes de información sobre una práctica sin reclamación & 35 & $\mathbf{1 , 1}$ \\
\hline Asuntos rechazados & 1051 & \\
\hline Con solicitud de alegaciones & $\mathbf{1 . 6 8 8}$ & $\mathbf{5 5 , 3}$ \\
\hline Allanamientos & 311 & 10,2 \\
\hline Informes favorables al reclamante & 544 & $\mathbf{1 7 , 8}$ \\
\hline Informes favorables a la entidad & 653 & $\mathbf{2 1 , 4}$ \\
\hline Improcedencia de informe. Sin pronunciamiento & 180 & 5,9 \\
\hline Desistimientos & $\mathbf{1 0 0}$ & $\mathbf{3 , 3}$ \\
\hline Total & $\mathbf{3 . 0 5 2}$ & \\
\hline
\end{tabular}

Fuente: Banco de España (2002), Memoria del Servicio de Reclamaciones 2001, p.24.

\subsection{Las iniciativas relacionadas con las pautas de "buen gobierno"}

Los diversos escándalos financieros comentados, unidos a otros aspectos como el estallido de la denominada burbuja tecnológica, los engaños contables, los conflictos de intereses, etc, han llevado también a reavivar el interés por las buenas prácticas de gobierno empresarial. El buen gobierno de las empresas permite aumentar la confianza de los inversores en las empresas $y$, por lo tanto, tal y como ha indicado recientemente el subgobernador del Banco de España, juega un papel muy importante a largo plazo en el crecimiento y bienestar de la sociedad por sus efectos en el coste y la obtención de financiación para los proyectos de inversión de las empresas.

Esta importancia es la que ha dado lugar a que en España, como en otros países, estén en marcha iniciativas legislativas y nuevos códigos de buen gobierno que intentan contribuir a mejorar dicho gobierno. Además de estas propuestas (aún sin plasmar en una norma promulgada en el caso español), encontramos otras iniciativas que proponen la aceptación voluntaria de ciertas pautas de buen gobierno. En este sentido, tenemos en primer lugar, el denominado Código Olivencia, que no se limita a las empresas financieras; 
más reciente, del año 2003, es el llamado Informe Aldama, que se refiere a los mercados financieros y a las sociedades cotizadas.

El primero de los citados, surgió en febrero de 1998 como resultado de una comisión que, presidida por Manuel Olivencia, se creó por el Consejo de Ministros en febrero de 1997, para redactar un informe sobre la problemática de los consejos de administración de las sociedades que apelan a los mercados financieros y para elaborar un código ético de buen gobierno de asunción voluntaria de estas sociedades. Las principales recomendaciones de este código se han recogido en el esquema 1 y, como se puede apreciar, en él, entre otros aspectos, se recomiendan actuaciones como la participación de consejeros independientes en los órganos de gobierno y la publicidad de las retribuciones de los altos cargos. A pesar de su interés, en general, su aplicación ha sido poco satisfactoria.

En cuanto al Informe Aldama, ha sido el resultado de una comisión creada también por el Consejo de Ministros con el objeto de analizar el estado y el grado de aplicación del código de buen go bierno de las sociedades cotizadas (el código Olivencia), así como para "estudiar los criterios y las pautas a las que deben someterse las sociedades emisoras de valores e instrumentos admitidos a negociación en los mercados organizados, (...) con la finalidad de aumentar la transparencia y la seguridad en los mercados financieros"12.

Según indica el propio informe, en el año 2002 no se sabía con precisión hasta qué punto había sido aplicado el código de buen gobierno; además, se había constatado que la opinión mayoritaria de los expertos y los accionistas era que, en la práctica, algunas de las propuestas de dicho código no se cumplían y existía una falta de transparencia que permitía los conflictos de intereses y el uso de información privilegiada por parte de directivos y consejeros en detrimento de las sociedades; por otro lado, aunque se veía con interés la figura del consejero independiente, se dudaba de que realmente tales consejeros fueran realmente independientes. Ante estas circunstancias, el Informe Aldama trata varios de los problemas abordados por la comisión presidida por Manuel Olivencia y otros nuevos, los cuales, junto con al gunas de las recomendaciones planteadas, también se han recogido en el esquema 1 de forma resumida.

En general, el Informe intenta buscar un equilibrio entre la regulación que va orientada a la protección de los derechos de los accionistas y, por otro

12 Acuerdo del Consejo de Ministros de 19 de julio de 2002. 


\section{ESQUEMA 1 \\ Principales recomendaciones del Código Olivencia y del Informe de la Comisión Aldama}

\begin{tabular}{|c|c|}
\hline & \\
\hline \multirow{6}{*}{ 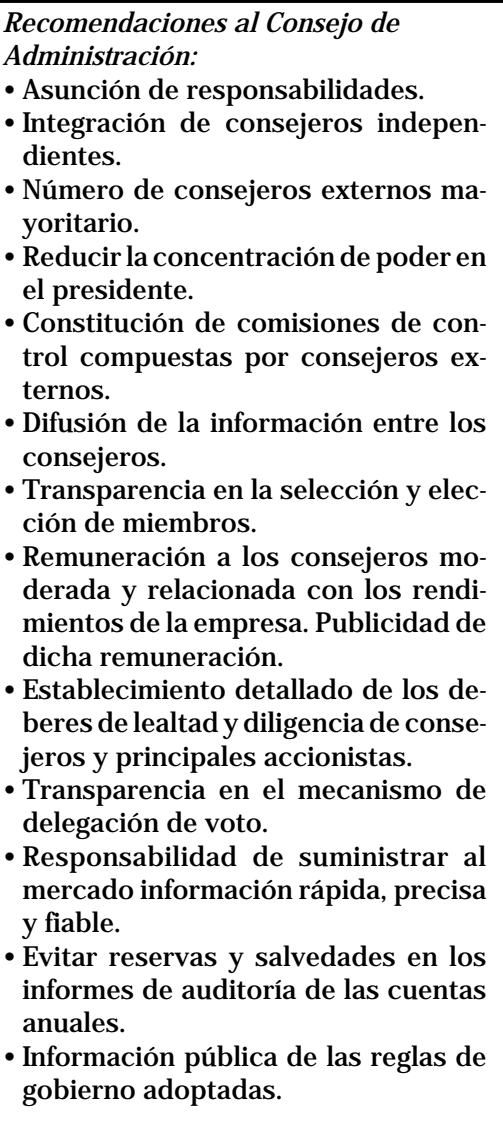 } & $\begin{array}{l}\text { deber de Intormacion: } \\
\text { ormación a las estruc- } \\
\text { no de las sociedades. } \\
\text { litar una información } \\
\text { ativa, simétrica y en }\end{array}$ \\
\hline & $\begin{array}{l}\text { tad. } \\
\text { ores: } \\
\text { especifique } \\
\text { inistradores, } \\
\text { cativos, etc. } \\
\text { las con el de- } \\
\text { o y esfuerzo } \\
\text { cuerdos con- } \\
\text {. }\end{array}$ \\
\hline & \\
\hline & $\begin{array}{l}\text { le la } \\
\text { lón y } \\
\text { los }\end{array}$ \\
\hline & Oli- \\
\hline & $\begin{array}{l}\text { Recomendaciones para mejorar la fiabilidad y exac- } \\
\text { titud de los informes de profesionales externos ( ana- } \\
\text { listas financieros, bancos de inversión y agencias de } \\
\text { calificación) como medio de proteger a los inverso- } \\
\text { res y accionistas. }\end{array}$ \\
\hline
\end{tabular}

Fuente: Elaboración propia a partir de:

(1998), Código de buen gobierno de las sociedades cotizadas y (2003), Informe de la Comisión especial para el fomento de la transparencia y seguridad en los mercados y en las sociedades cotizadas. 
lado, la mayor autorregulación posible de las empresas cotizadas. Por ello, se afirma en él que es necesario reforzar el ejercicio de la libertad de los accionistas y de la autorregulación de las empresas para decidir y aplicar sus estrategias, así como organizar su gobierno corporativo; según otras opiniones, el contenido del Informe confirma la necesidad de una legislación. Una norma, que tiene su origen en el Código Olivencia, obliga a las sociedades cotizadas a la creación de un comité de auditoría.

\subsection{Las iniciativas del sector privado}

Los escándalos descritos en la primera parte tenían necesariamente que llevar también a cambios en los comportamientos bancarios, aparte de las posibles implicaciones judiciales. Efectivamente, algunas de las propuestas comentadas previamente han sido llevadas a cabo por ciertas entidades. Así por ejemplo, los dos grandes bancos han aprobado códigos debuen gobierno y han elaborado informes de responsabilidad social, incorporando criterios medioambientales a sus inversiones. En este sentido, el Santander Central Hispano ha presentado un Plan de Responsabilidad Social Corporativa en el que se compromete con la transparencia, el buen gobierno, la calidad, la gestión de recursos humanos y el desarrollo socioeconómico, además de fomentar la protección del medio ambiente; todos estos aspectos se incorporan a los diferentes niveles de la organización desde el punto de vista de la estrategia y de la gestión.

Además de lo anterior, se ha ampliado la información a los inversores (destacan en este sentido BSCH y Bankinter); se han establecido moderaciones en los sueldos de los consejeros y se han reforzado sus incompatibilidades; la mayor parte de las entidades de crédito han creado la figura del defensor del cliente para atender las reclamaciones ${ }^{13}$; $y$ en algún caso se han suprimido las medidas antiblindajes que trataban de evitar OPAS hostiles, pero que afectaban negativamente a los derechos de los pequeños accionistas. Evidentemente, la virtualidad de estas medidas depende desu aplicación

13 La ley financiera, de reciente aprobación, obliga a que las entidades de crédito, aseguradoras y empresas de servicios de inversión cuenten con un servicio o departamento de atención al cliente para atender y resolver las quejas de los usuarios. Este servicio no será incompatible con la figura del defensor del cliente, que habrá de ser una persona independiente y de reconocido prestigio, la cual debe atender y resolver las reclamaciones dispuestas en su reglamento de funcionamiento y promover el cumplimiento de la normativa de transparencia y protección de la clientela. 
futura y, sobre todo, del cambio de comportamiento tradicional de las entidades.

También como respuesta de la sociedad civil han aparecido las asociaciones de consumidores; en concreto en el terreno financiero, existe la Asociación de Usuarios de Servicios Bancarios (AUSBANC). En el ámbito internacional, los grandes inversores institucionales (fondos de inversión y de pensiones) se han reunido para definir las medidas para promover de forma conjunta las prácticas de buen gobierno de las empresas para evitar que se vuelvan a evitar escándalos empresariales que han supuesto fuertes pérdidas en el valor de sus carteras. Todas éstas son iniciativas, no exentas de críticas, que pueden ayudar a defender los derechos de los inversores y ahorradores ${ }^{14}$.

La preocupación sobre el destino de las inversiones financieras ha llevado también a la creación de otras iniciativas; por ejemplo, los fondos éticos y solidarios, que pretenden asegurar una inversión responsable de ciertos fondos de inversión y destinar recursos a ciertas actividades solidarias, respectivamente; Ios planes y convenios para asegurar recursos a las PYMES; y el sistema de microcréditos, dirigidos a empresas de muy pequeña dimensión, localizadas sobre todo en países el Tercer Mundo.

\section{Conclusiones}

Hemos contemplado diversos comportamientos de los mercados financieros que ponen en cuestión si el sistema está cumpliendo adecuadamente sus funciones en beneficio de la economía española.

Podríamos apuntar varias causas que han originado estos hechos:

1) Un tratamiento muy escaso y muy benigno del delito económico y financiero.

2) El mayor poder económico de las instituciones financieras en relación con su clientela.

3) La liberalización del sistema financiero, especialmente en los años

14 Por ejemplo, algunas prácticas seguidas por las entidades financieras han sido objeto de resolución judicial y, posteriormente, han sido reguladas gracias a las denuncias presentadas por estas asociaciones (así ha sucedido recientemente con la práctica del redondeo al alza en la obtención del tipo de interés a aplicar en los préstamos a interés variable). 
setenta, que eliminó, de forma adecuada, las restricciones al funcionamiento de las entidades, pero que también afectó negativamente a la supervisión y el control.

Las múltiples reacciones que han provocado estos problemas se pueden clasificar en dos grupos:

1) Iniciativas de autorregulación, apoyadas sobre todo por los defensores de la liberalización de la actividad económica, que pretenden que sean los mercados los que valoren y sancionen los comportamientos incorrectos.

2) La aprobación de normas jurídicas que sancionen estos comportamientos y que establezcan claramente las fronteras entre lo legal y lo ilegal.

Es cierto que no se puede judicializar los mercados y que no se puede olvidar que existen casos que escapan fácilmente a los procedimientos judiciales. Hay que subrayar también que algunos procedimientos son aceptables desde la norma pero pueden causar daños al mercado. Nuestra opinión es que la autorregulación es necesaria, pero que ello no obsta para que existan normas que obliguen a un comportamiento prudencial de las entidades y que castiguen severamente las actividades ilegales.

\section{Bibliografía}

Argandoña, A., (Ed.), (1995), La dimensión ética de las instituciones y mercados financieros, Bilbao, Ed. Fundación BBV.

Baena Tobar, N., (2002), La regulación del a buso de mercado en Europa y Estados Unidos, Colección Monografías, № 1, Madrid, Ed. CNMV.

Camacho Laraña, I., (1996), "Dimensión ética de las actividades financieras", Revista de Fomento Social, no 201, pp. 23-54.

Cuervo, A., (1995), La crisis bancaria en España 1977-1985, Barcelona, Ed. Ariel.

Fernández FernÁndez, J . L., (1994), "Sistema financiero y problemas morales: una agenda para la reflexión ética", Revista de Fomento Social, no 195, pp. 387-412.

López Caballero, A., (1995), “El problema ético de la información privilegiada. La nueva ley alemana", ICADE, no 35, pp. 93-110.

Rodero Franganillo, A., (1994), "El papel de la banca en la economía española" Revista de Fomento Social, no 196, pp. 565-582. 